inequalities can drive both the population level impact and the distributional impacts of policies is not well understood. In this study we use an existing health economic model of alcohol brief interventions (BIs) to explore how socioeconomic inequalities in different model inputs can affect conclusions about value for money and health inequality and contrast these with similar results for a smoking cessation model.

Methods BI policies were assessed using the Sheffield Alcohol Policy Model. Previous analysis has shown a national BI programme for alcohol to be both cost-effective and inequalityimproving. We examined whether these conclusions changed under three scenarios: i) individually excluding socioeconomic gradients in each model input, ii) raising levels of uptake to those in the 'best' group, iii) using different baseline populations. Impacts on total population health and health inequality were assessed using incremental population Net Health Benefit (NHB) and incremental 'Equally Distributed Equivalent' (EDE) health respectively. Results are compared with those from similar analyses undertaken using a smoking cessation model.

Results A national BI programme improved both health $(+43,016$ QALYs) and EDE (+50,792 QALYs), reducing health inequalities. Excluding gradients in model inputs had generally small effects on NHB $(+0 \%$ to $+10.4 \%)$ but a larger effect on $\operatorname{EDE}(-7.9 \%$ to $+15.7 \%)$, although not enough to change the conclusion that the policy is inequality reducing. Increasing delivery to the 'best' level would increase EDE to a greater extent than NHB $(+51.6 \%$ and $+43.5 \%$ respectively), further reducing inequalities.

Conclusion Unlike smoking cessation programmes, BIs are likely to be both cost-effective and reduce inequalities. Considering potential inequalities across all stages of intervention delivery is important when considering the impact of policies on health inequalities, even if it may not substantially affect decisions based solely on cost-effectiveness. The relative importance of socioeconomic gradients in different stages is likely to vary between risk factors and settings.

\section{OP61 HIERARCHICAL MODELS FOR INTERNATIONAL COMPARISONS: A CASE STUDY OF SMOKING, DISABILITY AND SOCIAL INEQUALITY IN 21 EUROPEAN COUNTRIES}

${ }^{1} G$ Disney*, ${ }^{2} \mathrm{~L}$ Gurrin, ${ }^{1} \mathrm{~A}$ Milner, ${ }^{1} \mathrm{~A}$ Kavanagh, ${ }^{1} \mathrm{Z}$ Aitken, ${ }^{3} \mathrm{D}$ Petrie. ${ }^{1}$ Centre for Health Equity, University of Melbourne, Melbourne, Australia; ${ }^{2}$ Centre for Epidemiology and Biostatistics, University of Melbourne, Melbourne, Australia; ${ }^{3}$ Centre for Health Equity, University of Melbourne, Melbourne, Australia

10.1136/jech-2019-SSMabstracts.63

Background International comparisons of social inequalities in health are challenging. The level of disaggregation often required can result in sparse data. We show the value of a hierarchical Bayesian approach that partially pools country-level estimates, reducing the influence of sampling variation and increasing the stability of estimates. A further challenge is how to simultaneously present the level of inequality and their precision on relative and absolute scales. We illustrate a new way of displaying estimates of prevalence, relative and absolute inequalities, and the uncertainty of these three estimates, on one plot. Methods We used the 2014 European Social Survey to estimate smoking prevalence, absolute and relative inequalities for men and women with and without disabilities in 21 European countries. Smoking prevalence estimates are generated from a hierarchical Bayesian model, where we assume country-specific estimates are drawn from an overall 'population distribution'. The model is set up with the likelihood (country-specific data) and priors (the assumed population distribution) pulling in opposite directions. The likelihood pulls the country-specific smoking prevalence estimate towards the observed value, whereas the priors pull the prevalence towards the European average, a phenomenon known as shrinkage. We simultaneously display smoking prevalence for people without disabilities (x-axis), absolute (y-axis) and relative inequalities (contour lines), capturing their uncertainty by plotting a 2-D normal approximation of the posterior distribution from the full probability (Bayesian) analysis.

Results Our model shifts more extreme prevalence estimates, based on fewer observations, toward the European average. For example disabled males in Portugal have a high observed smoking prevalence (53\%, 95\% Uncertainty Interval (UI): 42\%-63\%) and a small number of observations (23). The model shifts this estimate, shrinking it to $43 \%$ (UI 38\%-58\%). Conclusion We argue that our contributions allow for better decision making under uncertainty, through the combination of better statistical inference and an indication of the strength of evidence decision-makers have access to. Sensitivity to shrinkage could indicate whether a policy maker could benefit from more data to make an informed decision. If estimates are insensitive to partial pooling then a policy maker could be relatively confident in their evidence/data. Conversely, in a country where there is a large amount of shrinkage, decision making could still benefit from the collection of more data. Being able to include all this information on a single graphic provides a useful tool for evaluating both the geographical patterns of variation in, and strength of evidence for, differences in social inequalities in health.

\section{OP62 DOES SMOKING, DRINKING, PHYSICAL ACTIVITY, AND SELF-REPORTED HEALTH MEDIATE THE RELATION BETWEEN EDUCATION AND VOTING DURING MIDLIFE? EVIDENCE FROM THE 1958 NATIONAL CHILD DEVELOPMENT STUDY}

${ }^{1,2} \mathrm{~T}$ Gagné ${ }^{*},{ }^{1,3}$ I Schoon, ${ }^{1,2} \mathrm{~A}$ Sacker. ${ }^{1}$ International Centre for Lifecourse Studies in Society and Health, University College London, London, UK; ${ }^{2}$ Research Department of Epidemiology and Public Health, University College London, London, UK; ${ }^{3}$ Institute of Education, University College London, London, UK

10.1136/jech-2019-SSMabstracts.64

Background Education is a strong predictor of voting in most Western countries. New studies, predominantly from the United States, question the role of health as a mechanism reinforcing social inequalities in voting over the life-course. In the United Kingdom, we previously found that heavy smoking, physical inactivity, poor self-reported health, and, to a lesser extent, drinking over the recommended limit were each associated with a lower probability of voting in the 1958 National Child Development Study (NCDS). Building on these findings, this study examines the proportion of the association between educational attainment and voting that is mediated through these health indicators.

Methods We used the data of 6,166 NCDS participants who responded to each sweep between the ages of 23 and 50. We examined educational attainment at the age of 23, smoking, drinking, physical activity, self-reported health at the ages of 23, 32, and 42, and voting behaviour at the age of 42, 46, and 50. Adjusting for non-response and attrition using inverse- 
probability weights and missingness using multiple imputation, we compared prevalence ratios to examine: 1) the direct effect of having a higher level of education on voting when accounting for these health indicators, and 2) the proportion of its total effect that is eliminated by this adjustment.

Results Contrasting extreme groups, we found that participants with a degree (NVQ5/6) at the age of 23 were 25\%, 28\%, and $32 \%$ more likely to have voted in the last general election compared to those with no qualifications at the ages of 42, 46, and 50. Adjusting for health indicators at the ages of 23, 32, and 42, participants with a degree remained $19 \%$, $24 \%$, and $27 \%$ more likely to have voted at the ages of 42 , 46 , and 50, respectively. This translates into an average proportion eliminated of $22 \%, 18 \%$, and $16 \%$ across these age points. Testing mediators separately, we found that smoking, physical activity, and self-reported health were each likely to contribute to the 'education-voting' association.

Discussion In keeping with health promotion principles, health represents beyond the absence of disease a resource for individuals, their social network, and their communities. Our findings suggest that health and its behavioral determinants are likely to explain a portion of social inequalities in voting over the life-course. Research and intervention should address the specific health-related mechanisms through which current electoral processes may unequally influence voter turnout across social groups.

\section{OP64 THE IMPACT OF POLITICAL ECONOMY ON POPULATION HEALTH: A SYSTEMATIC REVIEW OF REVIEWS}

${ }^{1} \mathrm{G}$ McCartney*, ${ }^{1}$ W Hearty, ${ }^{1} \mathrm{~J}$ Arnot, ${ }^{2} \mathrm{~F}$ Popham, ${ }^{3} \mathrm{~A}$ Cumbers, ${ }^{3} \mathrm{R}$ McMaster. ${ }^{1} \mathrm{NHS}$ Health UK; ${ }^{2}$ CSO/MRC SPHSU, University of Glasgow, Glasgow, UK; ${ }^{3}$ University of Glasgow, Glasgow, UK

10.1136/jech-2019-SSMabstracts.65

Background Although there are many studies considering specific aspects of political economy and health, there have been few attempts to synthesise the literature. This work describes a systematic review of reviews of the literature describing the impact of political economy on population health.

Methods We searched Medline, Embase, International Bibliography of the Social Sciences (IBSS), Proquest Public Health, Sociological Abstracts, Applied Social Sciences Index and Abstracts (ASSIA), EconLit, SocIndex, Web of Science and the grey literature via 'Google Scholar'; for reviews of the literature. Relevant exposures were differences or changes in: policy, law or rules; economic conditions; institutions or social structures; politics, power or conflict. Relevant outcomes were any overall measure of population health such as self-assessed health, mortality, life expectancy, survival, morbidity, wellbeing, illness, ill-health and lifespan. All citations were reviewed independently by two authors for relevance. Critical appraisal of all included reviews was undertaken using modified AMSTAR criteria and then synthesised narratively giving greater weight to the higher quality reviews.

Results From 4,912 citations, 58 reviews were included. Both the quality of the reviews and the underlying studies within the reviews were variable. Social democratic welfare states, higher public spending, fair trade policies, extensions to compulsory education provision, microfinance initiatives in low income countries, health and safety policy, improved access to healthcare, and high quality affordable housing have positive impacts on population health. Neoliberal restructuring seems to be associated with increased health inequalities and higher income inequality with lower self-rated health and higher mortality. There are evidence gaps on the relationship between governance, polities, power, macroeconomic policy, public policy and population health, including the social class processes and forms of discrimination which generate inequalities. For some areas, such as the relationship between income inequality and mean population mortality, there is a need for a high quality systematic review. Primary research gaps also existing, for example on the impact of housing policy, availability and tenure.

Conclusion Politics, economics and public policy are important determinants of population health. Countries with social democratic regimes, higher public spending and lower income inequalities have populations with better health. There are substantial gaps in the synthesised evidence on the relationship between political economy and health and there is a need for higher quality reviews and empirical studies in this area. However, there is sufficient evidence in this review, if applied through policy and practice, to have marked beneficial health impacts.

\section{Obesity \& Physical Activity}

\section{OP65 THE EFFECTS OF PEDOMETER AND OTHER STEP-COUNT MONITORING INTERVENTIONS ON PHYSICAL ACTIVITY: A SYSTEMATIC REVIEW AND META-ANALYSIS OF RANDOMISED CONTROLLED TRIALS}

UAR Chaudhry*, C Wahlich, R Normansell, R Knightly, D Cook, T Harris. Population Health Research Institute (PHRI), St George's University of London (SGUL), London, UK

\subsection{6/jech-2019-SSMabstracts.66}

Background Physical inactivity is a growing public health concern, and the fourth leading cause of death globally. Pedometers measure step-counts and can increase physical activity levels. Newer devices, for example mobile phone applications and body worn devices, also measure step-counts and require scrutiny of their effectiveness. Our primary aim is to conduct a systematic review and meta-analysis of the effects of pedometer and other step-count monitoring interventions on physical activity levels among the adult general population.

Methods We systematically searched seven databases using $\mathrm{MeSH}$ headings and keywords to identify randomized controlled trials published after $1 / 1 / 2000$. We included trials with healthy adults participants aged $\geq 18$, or those at risk of disease. Children, those selected with a specific health condition, high-performance trainers and hospital-based studies were excluded. The intervention group comprised community-based step-count monitoring interventions including pedometers with objective physical activity measures; the comparator group incorporated 'usual standard care' or healthcare advice with minimal active engagement. The primary outcome was change in step-count at follow-up compared to baseline. A randomeffects model was utilized to assess the primary outcome, and a risk of bias assessment determined the quality of included studies. The protocol is registered PROSPERO: CRD42017075810. Results Following initial database searching of 14,356 records and subsequent forward citation search, 54 studies were included, of which 13 were part of the narrative synthesis. 41 studies were therefore incorporated in the quantitative metaanalysis; 22 providing estimated mean between-group 